\title{
5 Begleitende Evaluation des Freiburger Elterntrainings
}

\author{
Bettina Brehm, Judith E. Schill, Monica Biscaldi, Christian Fleischhaker
}

Zur Evaluation des FETASS werden die Eltern von Kindern mit Autismus-Spektrum-Störungen mit mehreren Fragebögen vor und nach dem Training befragt. Die Fragebögen werden vor und nach dem Elterntraining ausgegeben und müssen zu den Zeitpunkten nach der ersten und letzten Sitzung ausgefüllt werden:

Hierfür werden die folgenden Evaluationsmaße verwendet:

- Inventar zur Erfassung der Lebensqualität (ILK)

- Skala zur Erfassung sozialer Reaktivität (SRS)

- Elternstressfragebogen (ESF)

\subsection{Inventar zur Erfassung der Lebensqualität (ILK)}

Die ILK-Elternversion (Mattejat \& Remschmidt 2006) ist ein Screening-Instrument zur Erfassung der Lebensqualität bei Gesunden sowie Kindern und Jugendlichen mit einer psychischen Störung oder körperlichen Erkrankung und eignet sich zur Therapieevaluation.

\subsection{Skala zur Erfassung sozialer Reaktivität (SRS)}

Die Skala zur Erfassung sozialer Reaktivität (SRS) (Bölte \& Poustka 2008) ist ein Fremdbeurteilungsinstrument für Eltern zur Einschätzung der Symptomatik ihrer Kinder mit ASS. Die Eltern beurteilen ihre Kinder in 65 Items auf den folgenden 5 Skalen:

1. Soziale Bewusstheit

2. Soziale Kognition

3. Soziale Kommunikation

4. Soziale Motivation

5. Autistische Manierismen

\subsection{Elternstressfragebogen (ESF)}

Der Elternstressfragebogen (Domsch \& Lohaus 2010) ist ein Selbsteinschätzungsinstrument für Eltern mit 38 Items zur Beurteilung ihres aktuell wahrgenommenen Stresserlebens. Die Items sind jeweils auf einer vierstufigen Anwortskala zu bearbeiten. Es wird hier in der Regel die Form für das Grundschulalter verwendet.

Die Skalen des Elternstressfragebogens umfassen:

1. Elterliches Stresserleben

2. Rollenrestriktion

3. Soziale Unterstützung

4. Ggf. Partnerschaft 